\title{
Perirhinal and postrhinal, but not lateral entorhinal, cortices are essential for acquisition of trace eyeblink conditioning
}

\author{
Eugénie E. Suter, ${ }^{1}$ Craig Weiss, and John F. Disterhoft \\ Department of Physiology, Northwestern University Feinberg School of Medicine, Chicago, Illinois 60611, USA
}

\begin{abstract}
The acquisition of temporal associative tasks such as trace eyeblink conditioning is hippocampus-dependent, while consolidated performance is not. The parahippocampal region mediates much of the input and output of the hippocampus, and perirhinal (PER) and entorhinal (EC) cortices support persistent spiking, a possible mediator of temporal bridging between stimuli. Here we show that lesions of the perirhinal or postrhinal cortex severely impair the acquisition of trace eyeblink conditioning, while lateral EC lesions do not. Our findings suggest that direct projections from the PER to the hippocampus are functionally important in trace acquisition, and support a role for PER persistent spiking in time-bridging associations.
\end{abstract}

The behavioral paradigm of trace eyeblink conditioning (tEBC) is hippocampal-dependent in animals (Solomon et al. 1983; Moyer et al. 1990; Kim et al. 1995; Weiss et al. 1999a) and human data implicate awareness in tEBC acquisition (Clark and Squire 1998; Knuttinen et al. 2001), suggesting tEBC as a model for human declarative memory (Weiss and Disterhoft 2011). While tEBC acquisition is severely impaired with hippocampal lesions (Weiss et al. 1999a), consolidated retention is independent of the hippocampus (Kim et al. 1995). The Standard Consolidation Theory holds that hippocampal memories are transferred to extrahippocampal sites for long-term storage (Squire and Zola-Morgan 1991); other theories of consolidation also propose a greater involvement of extra-hippocampal sites in long-term or oft-revisited memories (Nadel and Moscovitch 1997; Winocur et al. 2010).

The rat parahippocampal region (PH), a homolog of the primate and human medial temporal lobe, comprises entorhinal (EC), perirhinal (PER), and postrhinal (POR) cortices and mediates much of the cortical input and output of the hippocampus (Fig. 1A; Scharfman et al. 2000; Furtak et al. 2007). The lateral EC (latEC), but not the medial EC (mEC), is required for the retention of tEBC (Morrissey et al. 2012), while the acquisition of tEBC depends on the mEC (Ryou et al. 2001), suggesting differential roles of PH cortices on this task across learning. Here, we tested the hypothesis that PER, POR, and latEC regions are critically important for the acquisition of tEBC.

The role of the hippocampus in tEBC may involve binding together the conditioned (CS) and unconditioned (US) stimulus across the "trace" period, the temporal gap between stimuli, since delay paradigms, in which CS and US are temporally contiguous, are not forebrain-dependent (Christian and Thompson 2003; Woodruff-Pak and Disterhoft 2008). Early human and animal studies of hippocampal lesions included damage to the PH (Mishkin 1982; Corkin et al. 1997) and later work showed that deficits in primates attributed to the hippocampus were in part due to PH destruction (Zola-Morgan et al. 1989). As input and output regions of the hippocampus, PH cortices have come under increased scrutiny and recent work has established time- and task-related requirements for PH subregions.

\footnotetext{
${ }^{1}$ Corresponding author

E-mail suter@northwestern.edu

Article is online at http://www.learnmem.org/cgi/doi/10.1101//m.028894.112.
}

The PER and POR are thought to subserve different pathways into and out of the hippocampus, with the POR and mEC continuing the "where" pathway from higher-order visual and other orienting stimuli, while the PER and latEC process "what" stream information (Eichenbaum et al. 2012). The PER receives multimodal sensory inputs and projects into the hippocampus mainly via the latEC, while the POR receives input mainly from visuospatial centers and projects to the mEC (Furtak et al. 2007). The hippocampal input in the CA1 and subiculum is segregated based on its origin in the PER/latEC or POR/mEC pathways (Naber et al. 1997), and the hippocampal output is similarly organized (Kloosterman et al. 2003b). Of note, in addition to heavy reciprocal projections with the EC, the main source of input to the hippocampus, both the PER and the POR also project directly to the hippocampus (Naber et al. 1999, 2001; Furtak et al. 2007). The functional importance of these (anatomically minor) pathways (Furtak et al. 2007), however, is not established.

The perirhinal and lateral entorhinal cortices both support persistent spiking in vitro (Egorov et al. 2002; Tahvildari et al. 2007; Navaroli et al. 2012), suggesting that these areas may support trace-bridging mechanisms independent of hippocampal function. Both the PER and latEC are essential for the acquisition of time-bridging trace fear (Kholodar-Smith et al. 2008; Bang and Brown 2009a; Esclassan et al. 2009). Receiving inputs at the PER from multiple higher-order sensory areas (Furtak et al. 2007), the PER and latEC are excellent candidates for a site that maintains recent inputs for association in the hippocampus proper after a temporal delay. In addition, the PER is essential in fear acquisition to discontinuous tones, which may be a function of auditory "object recognition" or of temporal tracking similar to that in trace tasks (Bang and Brown 2009b). A function for the PER in associative conditioning is supported by in vitro work, where the PER responds with universal depression to unpaired, but potentiates to paired cortical stimuli (Unal et al. 2012).

The temporal gradient of the hippocampus dependence in forebrain-dependent tasks (Kim et al. 1995) raises the question of whether the role of $\mathrm{PH}$ areas in acquisition and consolidation resembles that of the hippocampus, or parallels the post-consolidation recruitment of cortical areas (Squire and Zola-Morgan 1991; Nadel and Moscovitch 1997; Winocur et al. 2010). Ryou et al. (2001) showed impaired tEBC acquisition and decreased hippocampal task-related activity following entorhinal lesions centered on the mEC. In rats, retention of $\mathrm{tEBC}$ is dependent on 

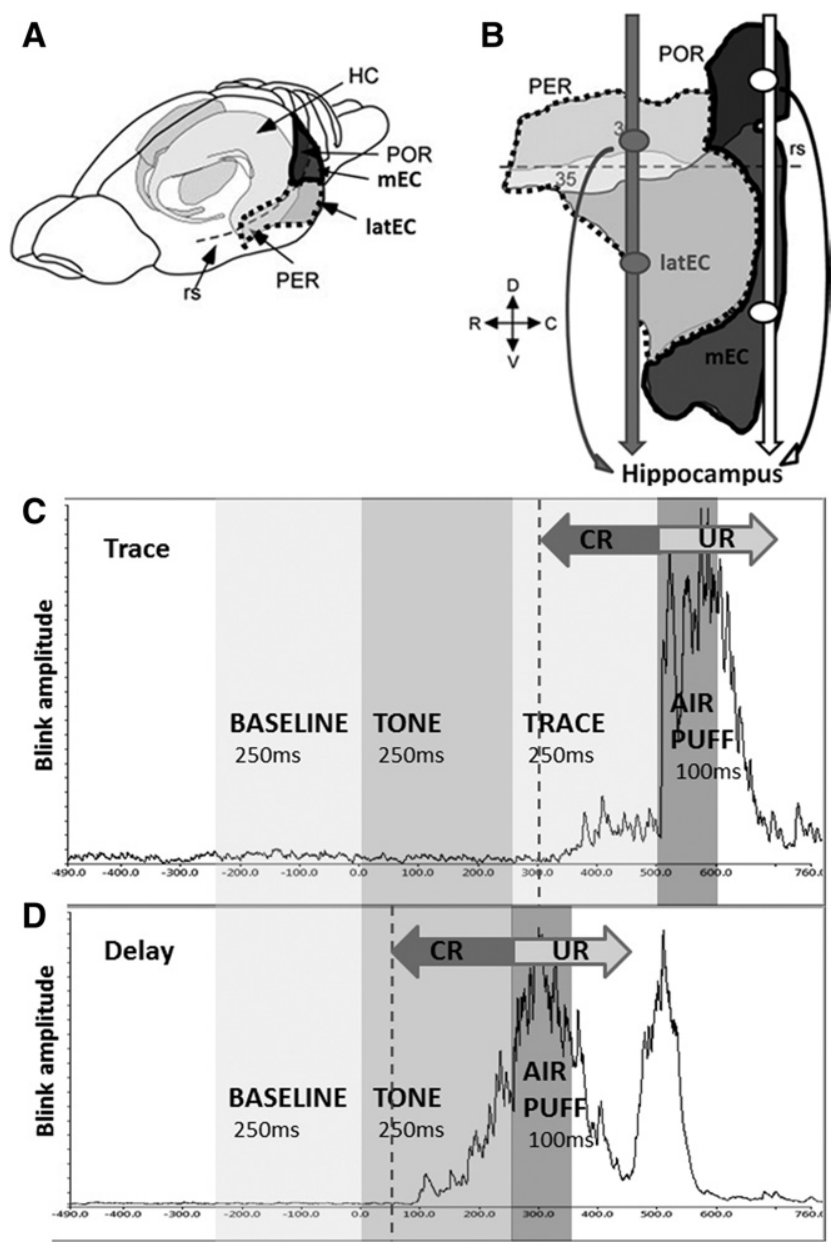

Figure 1. Parahippocampal cortices and eyeblink conditioning paradigm. (A) Anterolateral view of the rat brain showing subregions of the parahippocampal gyrus (adapted from Furtak et al. 2007 with permission from John Wiley and Sons (C) 2007). (B) Parahippocampal area unfurled with major projections indicated by arrows (adapted from Furtak et al. 2007 with permission from John Wiley and Sons (C) 2007). (C,D) EBC trial sequence with an example of a well-learned eyeblink conditioned response $(C R) ;(C)$ trace $=250 \mathrm{msec},(D)$ delay $($ trace $=0 \mathrm{msec})$. Dotted line (200 msec prior to airpuff) indicates the onset of the CR period for data analysis. (HC) hippocampus, (rs) rhinal sulcus.

latEC function, but not on the mEC (Morrissey et al. 2012). We used selective electrolytic lesions to test the individual contributions of the PER, POR, and latEC to the acquisition of timebridging trace as well as to delay eyeblink conditioning.

Rats were trained on trace and delay EBC after at least a 5-d recovery from bilateral electrolytic or sham lesion surgery at the PER or latEC or POR. Male Fischer 344xBrown Norway rats of at least $3 \mathrm{mo}$ in age received bilateral lesions $(200 \mu \mathrm{A} \times 20 \mathrm{sec}$ or sham: no current $\times 20 \mathrm{sec}$ ) under isoflurane anesthesia, followed by attachment of a tethering apparatus to the skull, with two electromyogram leads implanted in the orbicularis oculi muscle as described previously (Weiss et al. 1999b), to allow recording in the freely moving rat. Controls included shams as well as rats with tether-only surgery, as no differences were seen in behavior among these groups. The tether-only controls ensured an adequate statistical power to detect a difference between groups on the delay paradigm.

The tasks consisted of eyeblink conditioning in a light- and sound-attenuating chamber, using a tone CS and airpuff US (Fig.
1C,D). One habituation session was given, followed by training twice daily with 30 trials per session. The experimenter was blind to the lesion status. Eyeblink responses were evaluated as follows: a conditioned response (CR) was defined as an increase in EMG activity for at least $15 \mathrm{msec}$ within the $200 \mathrm{msec}$ preceding US onset, with an amplitude at least four standard deviations above the mean of the baseline (250 msec preceding CS onset). Trials with excessive activity during the baseline were excluded by removing any trial whose mean baseline was more than two standard deviations above the session mean. Percentages of CRs for the final four sessions (after animals had reached a behavioral plateau) were analyzed with repeated measures ANOVA and Fisher's PLSD post-hoc test in StatView. Rats received nine or ten sessions $(5 \mathrm{~d})$ of trace EBC, followed by six sessions ( $3 \mathrm{~d}$ ) of delay EBC. Of the POR lesioned rats, three did not complete delay training due to technical difficulties. Following perfusion with $10 \%$ formalin, brains were cryoprotected in 30\% sucrose, sliced, and stained for histological classification with Prussian Blue and Cresyl Violet.

Lesions were verified histologically for the inclusion of rats in the PER, POR, latEC, or sham lesion groups (Fig. 2, left column). Behavior was analyzed by comparing percent CR (mean \pm SEM) for each training session. Repeated measures ANOVA of the final four sessions of conditioning (trace, 6-9; delay, 3-6), showed a significant impairment of a lesion group on trace conditioning $\left(F_{(3,18)}=5.10, P<0.01\right.$; Fig. 2 , center column), but not on delay conditioning $\left(F_{(3,15)}=1.28, P>0.05\right.$; Fig. 2 , right column). All groups acquired delay EBC to control levels. Fisher's PLSD showed a significant impairment of both the PER and the POR groups on trace EBC as compared to controls (PER: $P<0.05$, POR: $P<0.01$; Fig. 2A,B). No statistical difference was seen between the PER and POR groups. The group with lesions of the latEC acquired trace EBC to control levels, with no statistical difference between groups (P>0.05; Fig. 2C).

We found that destruction of the PER or of the POR selectively prevents the acquisition of trace $\mathrm{EBC}$, a forebrain- and hippocampus-dependent task, with no effect on delay EBC. Although the latEC carries PER impulses to the hippocampus, bilateral destruction of the latEC did not impair tEBC. Ryou et al. (2001) showed impaired tEBC acquisition following entorhinal lesions centered on the mEC. While the largest lesions in that study extended into the latEC, the smallest lesions were selective for the $\mathrm{mEC}$, suggesting the $\mathrm{mEC}$ damage was responsible for the observed deficit found by Ryou et al. (2001). This conclusion is confirmed by our current finding that latEC-only lesions do not duplicate the deficit found by Ryou et al. (2001) with (medial) EC lesions. These findings are summarized in Figure 3: in acquisition (panel A), the latEC is not essential, whereas the PER, POR, $\mathrm{mEC}$, and hippocampus are required; while in retention (panel $\mathrm{B})$, the hippocampus and $\mathrm{mEC}$ are not essential, whereas the latEC is required. The PER and POR have not been tested post-acquisition.

The current investigation shows no difference between PER and POR involvement in trace EBC. Despite a clear separation of connectivity (Furtak et al. 2007) and function on a number of tasks that rely on spatial (POR) or temporal (PER) processing (Norman and Eacott 2005) or visual complexity (PER) (Murray et al. 2007), the POR and PER share strong anatomical interconnectivity (Furtak et al. 2007), and both the POR and PER are required for contextual fear acquisition (Bucci et al. 2000). This suggests that in non-spatial tasks such as tEBC, both the POR and PER may subserve task acquisition.

It has been suggested that time may be tracked in a manner analogous to space within the PH-hippocampus system (Martina et al. 2001; Gorchetchnikov and Grossberg 2007). Persistent spiking behavior and requirement in time-bridging tasks suggest a role 

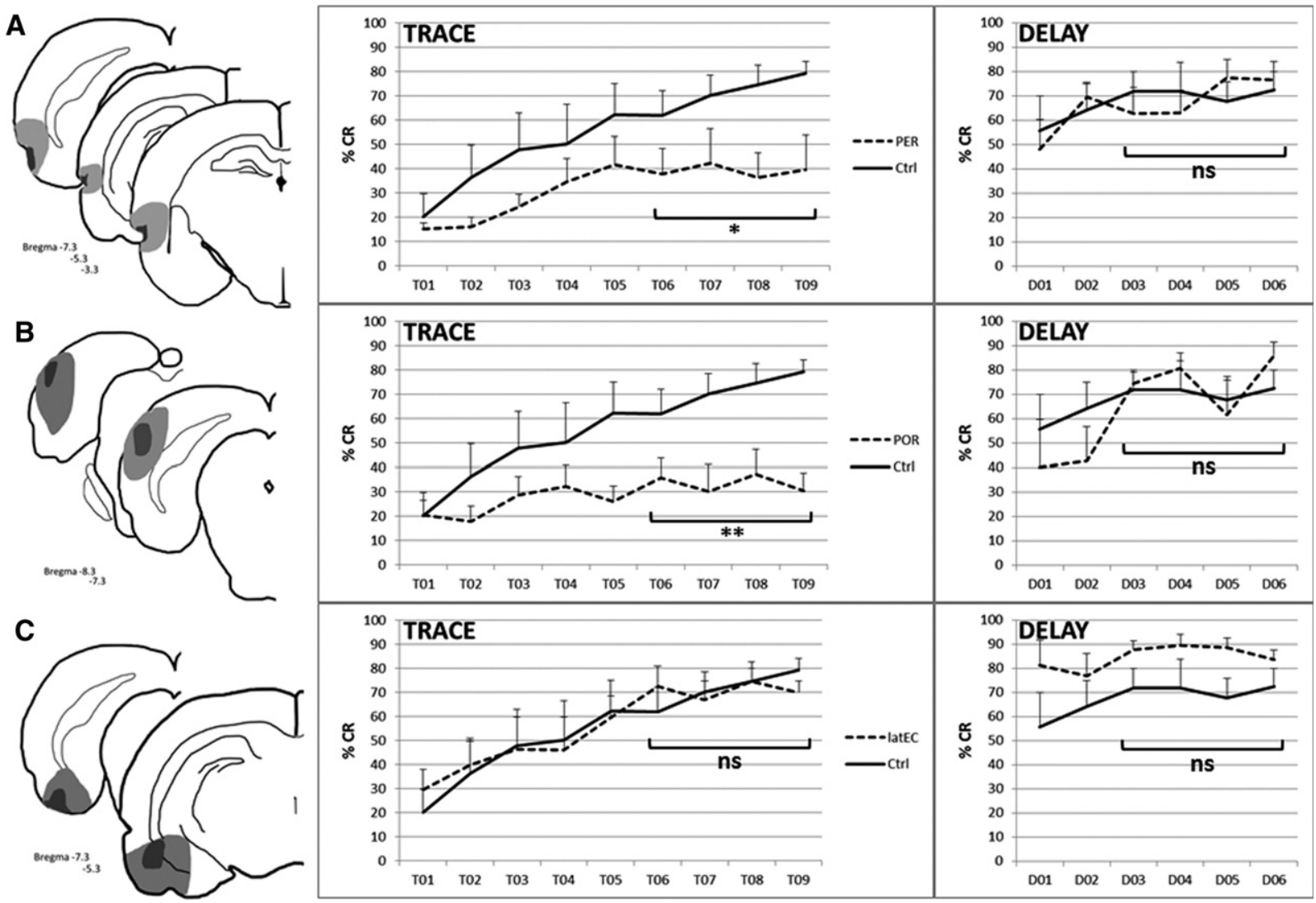

Figure 2. Histology (left column) and behavior are shown for each lesion group. (A) PER $(N=6),(B)$ POR $(N=6$, delay $N=3),(C)$ latEC $(N=4)$. Controls $(N=6)$. Light gray shading indicates the maximal and dark gray the minimal extent of the lesions. All the lesions were bilateral. Rats underwent training on trace $E B C$, followed by delay EBC. Data represent mean \%CR \pm SEM for each training session for trace (center column) and delay (right column) EBC. Results of repeated measures ANOVA comparing the lesion groups across the last four training sessions are indicated: $(* *) P<0.01,(*)$ $P<0.05$, ns $>0.05$ by Fisher's PLSD.

for PER-latEC in temporal navigation, but POR-mEC also shows potential as a timing circuit, due to the periodicity and oscillations demonstrated in spatial navigation (Hasselmo and Brandon 2008). We had hypothesized that a difference in tEBC lesion effects could elucidate which $\mathrm{PH}$ pathway functions in bridging time, but we find that tEBC relies on both PER and POR functions.

Interestingly, latEC lesions show a different pattern: we see no effect on trace EBC, even though trace fear acquisition is impaired by M1 antagonist pirenzepine at the latEC (Esclassan et al. 2009). Key differences to the current study include both the fear element of the task and the type of lesion, and render the data easily reconcilable. The latEC is heavily interconnected with lateral amygdala (Furtak et al. 2007), suggesting that fear circuitry is likely to affect latEC function. Additionally, the use of an M1 antagonist suggests a disruption of function, which can be more deleterious to hippocampal learning than destructive lesions in the hippocampal circuit (Solomon et al. 1983).

Much of the literature emphasizes PER projections to the hippocampus via the latEC. There has been extensive discussion as to signal propagation via direct PER-to-hippocampus projections (Canning and Leung 1997; Liu and Bilkey 1997). Careful follow-up studies, using current sink analysis, conclude that the PER and POR project directly to CA1/subiculum, but do not join the perforant path targeting dentate gyrus (Naber et al. 1999, 2001). In addition, minimal propagation of neocortical stimulation from the PER to the latEC in vitro supports an EC-independent associative role of the PER (Pelletier et al. 2004). Here we show that, while PER function is essential to $\mathrm{EBC}$ acquisition, the destruction of the latEC has no apparent effect on this task. In the absence of the latEC, essential PER input must reach the hippocampus via a different path, demonstrating that the anatomically minor PER-to-hippocampus direct projection (Furtak et al. 2007) is functionally important, as indicated in Figure 3, A, by the black arrow connecting the PER and the hippocampus. Because we used electrolytic lesions, damage to fibers of passage must be considered in

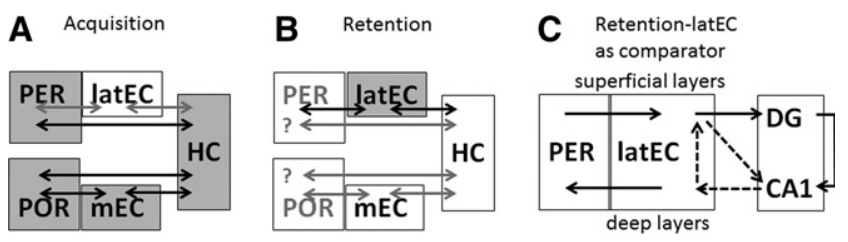

Figure 3. The direct projection from the PER to the hippocampus $(\mathrm{HC})$ is functionally important for $\mathrm{tEBC}$ acquisition. $(A)$ In acquisition, the latEC is not essential (white) whereas the PER, POR, $\mathrm{MEC}$, and $\mathrm{HC}$ are required. (B) For retention, the $\mathrm{HC}$ and $\mathrm{mEC}$ (white) are not essential, whereas the latEC is required; the PER and POR have not been tested post-acquisition. (C) Suggested function of the latEC in $\mathrm{EEBC}$ retention: at input in the (superficial) layers of the latEC, incoming information from the PER converges with hippocampal output from the deep latEC, forming a loop that may be used to compare current stimulus associations $(\mathrm{HC})$ with established associative memories (latEC). (DG) dentate gyrus. 
interpreting the results. If the deleterious effects of PER lesions were due to deafferentation of the latEC by damaging the fibers of passage, then latEC lesion results should mimic PER lesions, which is not the case.

In contradistinction to our acquisition results, previous studies showed that post-training inactivation of the latEC (using the GABA agonist muscimol) impaired the retention of tEBC (Morrissey et al. 2012). Of note, due to differences in the training schedules, the end of "acquisition" in the current study (day 5: latEC-induced deficit) precedes the onset of "retention" testing in Morrissey et al. (2012) (day 13: no deficit with a latEC lesion) by a week, leaving ample intervening time for memory consolidation processes that apparently account for the observed change in function between these studies. This temporal involvement pattern of the latEC is inverted as compared to that of the hippocampus, which is required for the acquisition, but not for the consolidated retention of tEBC (Kim et al. 1995). The double dissociation between the hippocampus and latEC lesions in the acquisition and retention of trace conditioning suggests that the latEC performs a consolidation-dependent associative role for previously learned time-bridging tasks. Long-term tEBC recall also shows a decreased functional connectivity between the hippocampus and EC, and increased functional connectivity between the EC and the putative storage node prefrontal cortex (Takehara-Nishiuchi et al. 2012), lending indirect evidence for a role for the EC in maintaining post-hippocampal associations. The PER and POR have not been examined in tEBC retention, but related tasks show PER or POR lesion-induced impairment on contextual fear conditioning acquisition with no decrease in impairment for as long as 100-d post-acquisition (Burwell et al. 2004).

The essential nature of the PER in tEBC suggests that persistent spiking mechanisms in this area (Navaroli et al. 2012), but not in the latEC, subserve trace-bridging during acquisition; the post-acquisition involvement of the latEC may reflect later recruitment of time-bridging machinery (Egorov et al. 2002) at a time when the hippocampus is no longer essential.

The latEC furthermore shows a striking pattern of segregation between input and output pathways, with input to the hippocampus arising from the superficial layers of the latEC, but output from the hippocampus projecting to the deep layers of the latEC. The additional flow of hippocampal output from the deep to the superficial EC suggests that the latEC may function as a comparator for hippocampal input and output (Kloosterman et al. 2003a). As such, the latEC's differential role in trace EBC acquisition vs. retention may reflect an increased importance either as a consolidated storage node for hippocampal associative memories, or as a moderator of acquired task performance. This circuit is illustrated in Figure 3, C, by dotted arrows.

In this study we show that tEBC acquisition depends on an intact PER and an intact POR, but not on the latEC, the main mediator of the PER input to the hippocampus. No difference was seen between the effects of the PER and POR lesions, despite functional segregation on other tasks. This suggests that direct projections from the PER to the hippocampal CA1/subiculum are functionally important in trace acquisition, and support a role for PER persistent spiking in time-bridging associations. In addition, our data suggest a role for the latEC specifically in consolidated, but not in the formation of, time-bridging associations. The latEC's increased role after acquisition, in combination with projections that combine hippocampal feedback with hippocampal input from the PER in the latEC's superficial layers, support the concept of the latEC as a monitor for associations formed by the hippocampus. In sum, these findings suggest that further investigation of the communication among the PER, latEC, and hippocampus during different stages of trace EBC is warranted.

\section{Acknowledgments}

We thank John M. Linardakis and Dr. James F. Baker for their assistance. This work was supported by the National Institutes of Health (NS059879 to C.W. and MH47340 to J.F.D.).

\section{References}

Bang SJ, Brown TH. 2009a. Muscarinic receptors in perirhinal cortex control trace conditioning. J Neurosci 29: 4346-4350.

Bang SJ, Brown TH. 2009b. Perirhinal cortex supports acquired fear of auditory objects. Neurobiol Learn Mem 92: 53-62.

Bucci DJ, Phillips RG, Burwell RD. 2000. Contributions of postrhinal and perirhinal cortex to contextual information processing. Behav Neurosci 114: $882-894$.

Burwell RD, Bucci DJ, Sanborn MR, Jutras MJ. 2004. Perirhinal and postrhinal contributions to remote memory for context. J Neurosci 24: $11023-11028$

Canning KJ, Leung LS. 1997. Lateral entorhinal, perirhinal, and amygdala-entorhinal transition projections to hippocampal CA1 and dentate gyrus in the rat: A current source density study. Hippocampus 7: 643-655.

Christian KM, Thompson RF. 2003. Neural substrates of eyeblink conditioning: Acquisition and retention. Learn Mem 10: 427-455.

Clark RE, Squire LR. 1998. Classical conditioning and brain systems: The role of awareness. Science 280: 77-81.

Corkin S, Amaral DG, Gonzalez RG, Johnson KA, Hyman BT. 1997. H. M.'s medial temporal lobe lesion: Findings from magnetic resonance imaging. J Neurosci 17: 3964-3979.

Egorov AV, Hamam BN, Fransen E, Hasselmo ME, Alonso AA. 2002. Graded persistent activity in entorhinal cortex neurons. Nature 420: 173-178.

Eichenbaum H, Sauvage M, Fortin N, Komorowski R, Lipton P. 2012. Towards a functional organization of episodic memory in the medial temporal lobe. Neurosci Biobehav Rev 36: 1597-1608.

Esclassan F, Coutureau E, Di Scala G, Marchand AR. 2009. A cholinergic-dependent role for the entorhinal cortex in trace fear conditioning. J Neurosci 29: 8087-8093.

Furtak SC, Wei SM, Agster KL, Burwell RD. 2007. Functional neuroanatomy of the parahippocampal region in the rat: The perirhinal and postrhinal cortices. Hippocampus 17: 709-722.

Gorchetchnikov A, Grossberg S. 2007. Space, time and learning in the hippocampus: How fine spatial and temporal scales are expanded into population codes for behavioral control. Neural Netw 20: 182-193.

Hasselmo ME, Brandon MP. 2008. Linking cellular mechanisms to behavior: Entorhinal persistent spiking and membrane potential oscillations may underlie path integration, grid cell firing, and episodic memory. Neural Plast 2008: 658323.

Kholodar-Smith DB, Allen TA, Brown TH. 2008. Fear conditioning to discontinuous auditory cues requires perirhinal cortical function. Behav Neurosci 122: 1178-1185.

Kim JJ, Clark RE, Thompson RF. 1995. Hippocampectomy impairs the memory of recently, but not remotely, acquired trace eyeblink conditioned responses. Behav Neurosci 109: 195-203.

Kloosterman F, Van Haeften T, Witter MP, Lopes Da Silva FH. 2003a. Electrophysiological characterization of interlaminar entorhinal connections: An essential link for re-entrance in the hippocampal-entorhinal system. Eur J Neurosci 18: 3037-3052.

Kloosterman F, Witter MP, Van Haeften T. 2003b. Topographical and laminar organization of subicular projections to the parahippocampal region of the rat. J Comp Neurol 455: 156-171.

Knuttinen MG, Power JM, Preston AR, Disterhoft JF. 2001. Awareness in classical differential eyeblink conditioning in young and aging humans. Behav Neurosci 115: 747-757.

Liu P, Bilkey DK. 1997. Current source density analysis of the potential evoked in hippocampus by perirhinal cortex stimulation. Hippocampus 7: 389-396.

Martina M, Royer S, Pare D. 2001. Propagation of neocortical inputs in the perirhinal cortex. J Neurosci 21: 2878-2888.

Mishkin M. 1982. A memory system in the monkey. Philos Trans R Soc Lond B Biol Sci 298: 83-95.

Morrissey MD, Maal-Bared G, Brady S, Takehara-Nishiuchi K. 2012. Functional dissociation within the entorhinal cortex for memory retrieval of an association between temporally discontiguous stimuli. J Neurosci 32: 5356-5361.

Moyer Jr. JRJr, Deyo RA, Disterhoft JF. 1990. Hippocampectomy disrupts trace eye-blink conditioning in rabbits. Behav Neurosci 104: 243-252.

Murray EA, Bussey TJ, Saksida LM. 2007. Visual perception and memory: A new view of medial temporal lobe function in primates and rodents. Annu Rev Neurosci 30: 99-122. 
Naber PA, Caballero-Bleda M, Jorritsma-Byham B, Witter MP. 1997. Parallel input to the hippocampal memory system through peri- and postrhinal cortices. Neuroreport 8: 2617-2621.

Naber PA, Witter MP, Lopez da Silva FH. 1999. Perirhinal cortex input to the hippocampus in the rat: Evidence for parallel pathways, both direct and indirect. A combined physiological and anatomical study. Eur J Neurosci 11: $4119-4133$.

Naber PA, Witter MP, Lopes da Silva FH. 2001. Evidence for a direct projection from the postrhinal cortex to the subiculum in the rat. Hippocampus 11: 105-117.

Nadel L, Moscovitch M. 1997. Memory consolidation, retrograde amnesia and the hippocampal complex. Curr Opin Neurobiol 7: 217-227.

Navaroli VL, Zhao Y, Boguszewski P, Brown TH. 2012. Muscarinic receptor activation enables persistent firing in pyramidal neurons from superficial layers of dorsal perirhinal cortex. Hippocampus 22: 1392-1404.

Norman G, Eacott MJ. 2005. Dissociable effects of lesions to the perirhinal cortex and the postrhinal cortex on memory for context and objects in rats. Behav Neurosci 119: 557-566.

Pelletier JG, Apergis J, Pare D. 2004. Low-probability transmission of neocortical and entorhinal impulses through the perirhinal cortex. J Neurophysiol 91: 2079-2089.

Ryou JW, Cho SY, Kim HT. 2001. Lesions of the entorhinal cortex impair acquisition of hippocampal-dependent trace conditioning. Neurobiol Learn Mem 75: 121-127.

Scharfman HE, Witter MP, Schwarcz R. 2000. The parahippocampal region. Implications for neurological and psychiatric diseases. Introduction. Ann N Y Acad Sci 911: x-xiii.

Solomon PR, Solomon SD, Schaaf EV, Perry HE. 1983. Altered activity in the hippocampus is more detrimental to classical conditioning than removing the structure. Science 220: 329-331.

Squire LR, Zola-Morgan S. 1991. The medial temporal lobe memory system. Science 253: $1380-1386$.
Tahvildari B, Fransen E, Alonso AA, Hasselmo ME. 2007. Switching between "on" and "off" states of persistent activity in lateral entorhinal layer III neurons. Hippocampus 17: 257-263.

Takehara-Nishiuchi K, Maal-Bared G, Morrissey MD. 2012. Increased entorhinal-prefrontal theta synchronization parallels decreased entorhinal-hippocampal theta synchronization during learning and consolidation of associative memory. Front Behav Neurosci 5: 90.

Unal G, Apergis-Schoute J, Pare D. 2012. Associative properties of the perirhinal network. Cereb Cortex. 22: 1318-2332.

Weiss C, Disterhoft JF. 2011. Exploring prefrontal cortical memory mechanisms with eyeblink conditioning. Behav Neurosci 125: 318-326.

Weiss C, Bouwmeester H, Power JM, Disterhoft JF. 1999a. Hippocampal lesions prevent trace eyeblink conditioning in the freely moving rat. Behav Brain Res 99: 123-132.

Weiss C, Knuttinen MG, Power JM, Patel RI, O'Connor MS, Disterhoft JF. 1999b. Trace eyeblink conditioning in the freely moving rat: Optimizing the conditioning parameters. Behav Neurosci 113: $1100-1105$.

Winocur G, Moscovitch M, Bontempi B. 2010. Memory formation and long-term retention in humans and animals: Convergence towards a transformation account of hippocampal-neocortical interactions. Neuropsychologia 48: 2339-2356.

Woodruff-Pak DS, Disterhoft JF. 2008. Where is the trace in trace conditioning? Trends Neurosci 31: 105-112.

Zola-Morgan S, Squire LR, Amaral DG, Suzuki WA. 1989. Lesions of perirhinal and parahippocampal cortex that spare the amygdala and hippocampal formation produce severe memory impairment. J Neurosci 9: 4355-4370.

Received October 3, 2012; accepted in revised form November 30, 2012. 


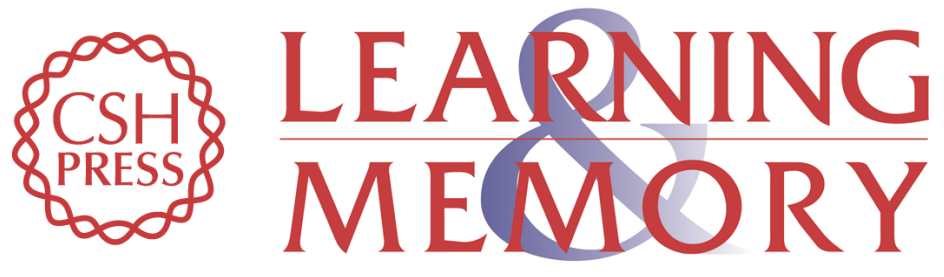

\section{Perirhinal and postrhinal, but not lateral entorhinal, cortices are essential for acquisition of trace eyeblink conditioning}

Eugénie E. Suter, Craig Weiss and John F. Disterhoft

Learn. Mem. 2013, 20:

Access the most recent version at doi:10.1101/lm.028894.112

References This article cites 45 articles, 11 of which can be accessed free at: http://learnmem.cshlp.org/content/20/2/80.full.html\#ref-list-1

License

Email Alerting Receive free email alerts when new articles cite this article - sign up in the box at the Service top right corner of the article or click here. 